STEM, STEAM, STREAM:

Posibilidades, reflexiones y experiencias

\title{
STEAM en Educación Infantil. Una visión desde las matemáticas
}

Recepción: 30/11/2020 | Revisión: 07/02/2021 | Aceptación: 06/08/2021 | Publicación: 01/10/2021

\author{
Montserrat PRAT \\ Universitat Blanquerna - Ramon Llull \\ montserratpm3@blanquerna.url.edu \\ https://orcid.org/oooo-00o2-8979-7663
}

\author{
Isabel SELLAS \\ Universitat de Vic \\ isabel.sellas@uvic.cat \\ http://orcid.org/oooo-0003-2200-569X
}

Resumen: Este artículo revisa diferentes propuestas STEAM para Educación Infantil, con el objetivo de analizar el papel que juegan en ellas las matemáticas. Se organizan las propuestas en tres grupos de actividades: las que ponen el foco en el pensamiento computacional, las que ponen el foco en la construcción y la experimentación, y finalmente, el grupo de propuestas con el foco en el "hazlo tú mismo". El análisis de estas actividades STEAM permite constatar que las matemáticas pueden jugar en ellas un papel instrumental o central, de manera similar al papel que desarrollan las matemáticas en la vida cotidiana. En todas las actividades STEAM examinadas tienen una importancia central tanto las capacidades matemáticas propias de Educación Infantil: identificar, comparar, clasificar, ordenar, entre otras; como los procesos matemáticos: resolución de problemas, representación, comunicación, conexiones y razonamiento. No obstante, la presencia de las matemáticas en las propuestas STEAM no asegura que el aprendizaje matemático se produzca de manera efectiva, pues este dependerá de la gestión que el adulto desempeñe mientras se realiza la actividad. Así, concluimos que, si queremos que las actividades STEAM produzcan el aprendizaje matemático esperado, debemos formar a los docentes para asegurar que sepan acompañar debidamente a sus alumnos en este proceso.

Palabras clave: STEAM; matemáticas; educación infantil; formación inicial del profesorado; interdisciplinariedad.

\section{STEAM IN CHILDHOOD EDUCATION. A VIEW FROM MATHEMATICS}

Abstract: This article reviews different STEAM proposals for Early Childhood Education, with the aim of analysing the role of mathematics. The proposals are organized into three groups of activities: those focusing on computational thinking, those focusing on construction and experimentation, and finally the group of proposals with "do it yourself" focus. The analysis of these STEAM activities shows that mathematics can play an instrumental or central role in them, similar to the role that mathematics plays in everyday life. In all STEAM activities examined, mathematics has an essential importance. Mathematics are key in these activities as mathematical abilities in Early Childhood Education: identifying, comparing, classifying, ordering, among others; but also, as mathematical processes: problem solving, representation, communication, connections, and reasoning. However, the presence of mathematics in the STEAM proposals does not ensure that mathematical learning 
occurs effectively. The mathematical learning will depend on the adult performance while the activity is carried out. Thus, we conclude that, if we want STEAM activities to produce the expected mathematical learning, we must train teachers to ensure that they know how to appropriately accompany their students in this mathematical learning process.

Keywords: STEAM; mathematics; childhood education; initial teacher training; interdisciplinarity.

STEAM EN EDUCACIÓ INFANTIL. UNA VISIÓ DES DE LES MATEMÀTIQUES

Resum: Aquest article revisa diverses propostes STEAM per a Educació Infantil, amb l'objectiu d'analitzar el paper que hi juguen les matemàtiques. Les propostes s'organitzen en tres grups d'activitats: les que posen el focus en el pensament computacional, les que posen el focus en la construcció i l'experimentació, i finalment, el grup de propostes amb el focus en el "fes-ho tu mateix". L'anàlisi d'aquestes activitats STEAM permet constatar que les matemàtiques poden jugar en elles un paper instrumental o central, de manera similar al paper que desenvolupen les matemàtiques en la vida quotidiana. En totes les activitats STEAM examinades hi tenen una importància central les capacitats matemàtiques pròpies de l'Educació Infantil: identificar, comparar, classificar, ordenar, entre altres; així com els processos matemàtics: resolució de problemes, representació, comunicació, connexions i raonament. No obstant això, la presència de les matemàtiques en les propostes STEAM no assegura que l'aprenentatge matemàtic es produeix de manera efectiva, ja que dependrà de la gestió que en faci l'adult mentre es desenvolupa l'activitat. Concloem que, si volem que les activitats STEAM produeixin l'aprenentatge matemàtic esperat, hem de formar els docents per assegurar que saben acompanyar els seus alumnes en aquest procés.

Paraules clau: STEAM; matemàtiques; educació infantil; formació inicial del professorat; interdisciplinarietat.

\section{Introducción}

El acrónimo STEM y STEAM se han hecho un hueco en el mundo educativo. Nació en los años 90 como una necesidad social para potenciar las vocaciones en ciencias (S), tecnología ( $T$ ), ingeniería (E) y matemáticas $(M)$, refiriéndose a las cuatro disciplinas separadamente, y no desde una perspectiva de educación integrada (Sanders, 2009). En la actualidad, en cambio, cuando pensamos en educación STEAM integrada las visiones varían, pero en ellas siempre aparece la concepción interdisciplinar de la educación, el aprendizaje basado en problemas, la resolución de problemas, el aprendizaje real y/o el trabajo en equipo.

La presencia de la A en el acrónimo STEAM propone una apuesta hacia la creatividad que, aunque es una capacidad intrínseca de las disciplinas STEM, se ve reforzada con la incorporación de la $A$ ya sea desde su significado más estricto (música, pintura, danza, etc.) o más amplio (bellas artes, expresión corporal, humanidades) (Yakman y Lee, 2012; Domènech-Casal, 2018). Esta apuesta por la creatividad es fundamental para la innovación (Ceschini, 2014).

En el contexto educativo actual se espera que los docentes enseñen de forma que desarroIlen las habilidades de aprendizaje del siglo XXI en sus alumnos. A pesar de ser diversos los marcos para el aprendizaje del siglo XXI, y a pesar de sus divergencias, todos coinciden en cuatro componentes: creatividad, colaboración, comunicación y pensamiento crítico (Beswick y Fraser, 2019). Los enfoques integrados STEAM ofrecen un entorno idóneo para desarrollar estas habilidades. 
Existen experiencias que abordan qué es la educación STEAM en Primaria, mientras que el número de experiencias y la fundamentación de cómo abordarla en Educación Infantil son menores. Asimismo, el estudio sobre educación STEAM dirigido al profesorado de primaria y secundaria, elaborado por Simarro y Couso (2018), identifica cuatro visiones de la educación STEAM: estética, globalizada, tecnocentrista e ingenieril. Estas autoras constatan que se trata de visiones que tienden a priorizar la A (arte), la T (tecnología), la E (ingeniería) o competencias transversales; y donde las ciencias y las matemáticas se presentan de manera aplicada como herramienta o como contexto.

Por todo ello, en este artículo nos proponemos revisar algunas de las aportaciones de la investigación sobre educación STEAM integrada en Educación Infantil y recopilar propuestas planteadas para esta etapa, con el objetivo de revisar el papel de las matemáticas en las actividades STEAM para las primeras edades, un papel que descubriremos clave y transversal. Dicha revisión también permitirá revisar el papel del docente para asegurar un aprendizaje matemático rico y adecuado en sus alumnos.

\section{STEAM en Educación Infantil}

En este apartado nos proponemos llevar a cabo una breve revisión teórica de la literatura existente acerca de STEAM, para centrarnos después en los resultados sobre experiencias STEAM en Educación Infantil. Se presentan estos contextos como oportunidades para el aprendizaje del alumnado de esta etapa, y también se analiza la realidad de los docentes para llevar a cabo estas experiencias, no siempre libres de dificultades.

\subsection{Una visión desde la investigación}

En el mundo educativo parece no existir una definición consensuada sobre qué es, cómo debe reflejarse en el currículum o desarrollarse en el aula la educación STEAM (Holmlund et al., 2018; Kelley y Knowles, 2016). Esto puede deberse, según Domènech-Casal (2018), a que se intenta dotar de significado didáctico y pedagógico a un término que en realidad es político. Así, para este autor, tiene sentido considerar todos los enfoques didácticos y pedagógicos que permitan alcanzar los objetivos STEAM. En este sentido, STEAM no solo es un modelo para la educación, sino que también es una forma de abordar la vida (Dziengel, 2018).

La educación STEAM integrada, en el sentido de Sanders (2009), explora el aprendizaje y la enseñanza de dos o más materias o áreas STEAM, y/o entre una materia STEAM y una o más materias escolares. La integración en todo caso debe superar la superposición de disciplinas (English, 2016; Rennie et al., 2017), es decir, ir más allá de tratarlas de forma independiente. En base a los estudios sobre el aprendizaje, la integración de las disciplinas favorece la comprensión de los conceptos integrados para transferirlos a situaciones nuevas, aunque también puede frenar el aprendizaje por la excesiva demanda de procesos cognitivos como atención y memoria de trabajo (NAENRC, 2014).

De los resultados de investigación se derivan tres implicaciones en el diseño de actividades STEAM: (i) la integración de las disciplinas debería ser explícita y dar soporte a los estudiantes para construir conocimiento, tanto en las disciplinas concretas como a través de las mismas, (ii) dar soporte a los alumnos para un uso adecuado del conocimiento específico de cada disciplina dado que el alumnado no siempre utiliza el conocimiento que tiene de cada una en contextos integrados, y (iii) tener en cuenta que no siempre más integración tiene porqué ser mejor (NAENRC, 2014). 


\subsection{La educación STEAM en Educación Infantil}

Si nos centramos en Educación Infantil, lo cierto es que la educación STEAM integrada parece tener mucha relación con el proceso de aprendizaje en esta etapa, pues se trata de una edad en la que nos gusta explorar y experimentar con el entorno (DeJarnette, 2018), resolver problemas y comparar cosas (Sharapan, 2012). Es una etapa donde las niñas y niños ven el mundo lleno de opciones y posibilidades infinitas (Jamil et al., 2018).

Existen investigaciones que abordan los beneficios que suponen la participación del alumnado de Educación Infantil en actividades STEAM, en este sentido McClure et al. (2017) señalan la correlación que existe entre las experiencias STEM integradas y el éxito posterior del alumnado en las asignaturas STEM.

Muchos docentes de Educación Infantil parecen tener dificultades para plantear actividades STEM que cubran las necesidades del alumnado de esta etapa (McClure et al., 2017), de hecho, la formación que reciben los docentes de Educación Infantil, y también en Primaria, se basa poco o casi nada en las disciplinas STEAM, a diferencia de la formación que reciben los maestros de secundaria (DeJarnette, 2018). Además, en general, se trata de una formación por contenidos específicos donde rara vez aprenden a trabajarlos de manera conjunta (Bennett y Ruchti, 2014). Esto conlleva que las maestras y los maestros de Educación Infantil puedan sentirse intimidados, inseguros o incluso puedan tener una predisposición negativa respecto a la educación STEAM integrada (Jamil et al., 2018).

Las escuelas de Educación Infantil y Primaria ofrecen un contexto ideal para generar situaciones de aprendizaje integrado, con periodos de tiempo flexibles para su desarrollo (Russo et al., 2011). No obstante, la realidad de la formación docente que han recibido estas maestras y maestros con poco conocimiento del contenido de las disciplinas STEAM dificulta que puedan generar situaciones de aprendizaje en dichos contextos. Desde el punto de vista matemático, una dificultad añadida para los docentes de Educación Infantil en las experiencias STEM y STEAM es la falta de conocimiento de las progresiones de aprendizaje en matemáticas, es decir, del conocimiento de los objetivos, de la secuencia del pensamiento y de las actividades y estrategias que permiten la progresión (McClure et al., 2017).

En este mismo sentido, si se espera que los docentes preparen a su alumnado para adquirir las competencias del siglo XXI, parece imprescindible que ellos mismos posean esta competencia, un hecho que parece haberse ignorado en gran medida hasta la fecha (Beswick y Fraser, 2019). Siendo necesario plantearse la importancia de las habilidades que debe poseer el maestro del siglo XXI (Beswick y Fraser, 2019). En definitiva, como señalan Simarro y Couso (2018), la visión y el conocimiento de los docentes es clave, pues es la que tiene más impacto en las aulas.

\section{Actividades STEAM para infantil}

Uno de los retos clave al plantear actividades STEAM para Educación Infantil consiste en describirlas de manera que no se identifiquen con hacer manualidades (Catchen, 2013). A pesar de que las actividades STEAM, en la mayoría de los casos, implican construir objetos o experimentar, van más allá de las manualidades en su búsqueda por integrar el currículum de manera transversal. 
Las actividades STEAM que encontramos en las escuelas, en libros especializados, en las experiencias compartidas por docentes, o en la investigación son diversas. En este trabajo se han organizado en tres grupos para poder presentarlas, entendiendo que al ser todas actividades STEAM tienen aspectos en común como la interdisciplinariedad, la manipulación o la experimentación. La organización que se presenta tiene en cuenta qué aspecto consideramos en este trabajo como foco principal de la propuesta o en cómo se presenta la propuesta en las fuentes consultadas. Así las propuestas STEAM para Educación Infantil las podemos agrupar en tres:

- Actividades con foco en el pensamiento computacional. En este grupo situaríamos actividades de robótica educativa y pensamiento computacional cómo las que usan Bee-bots (Ferrada et al., 2019; SteamCat, 2020), Cubetto (Caguana et al., 2017) o ScratchJr (Papadakis et al., 2016; Portelance et al., 2016).

- Actividades con foco en la experimentación y la construcción. Se trata de proyectos interdisciplinarios centrados en la experimentación y la construcción, propuestas de proyectos manipulativos para trabajar a partir de la resolución de problemas (Dziengel, 2018; SteamCat, 2020).

- Actividades con foco en el "hazlo tú mismo" (movimiento Maker). En este grupo encontramos los espacios de creación, los conocidos como makerspaces (Brejcha, 2018; Graves y Graves, 2016) y la metodología Tinkering (Simarro, 2019; SteamCat, 2020).

\subsection{Actividades con foco en el pensamiento computacional}

Los Bee-bots (de TTS), el Cubetto (de Prymo) o el ratón Jack (de Learning Resources) son tres ejemplos de propuestas que buscan introducir conceptos de pensamiento computacional en las primeras edades de una manera práctica e interactiva. Es habitual situar este tipo de materiales como propuestas STEAM pues abordan algunas de las letras del acrónimo: tecnología $(T)$, ingeniería $(E)$ y matemáticas $(M)$.

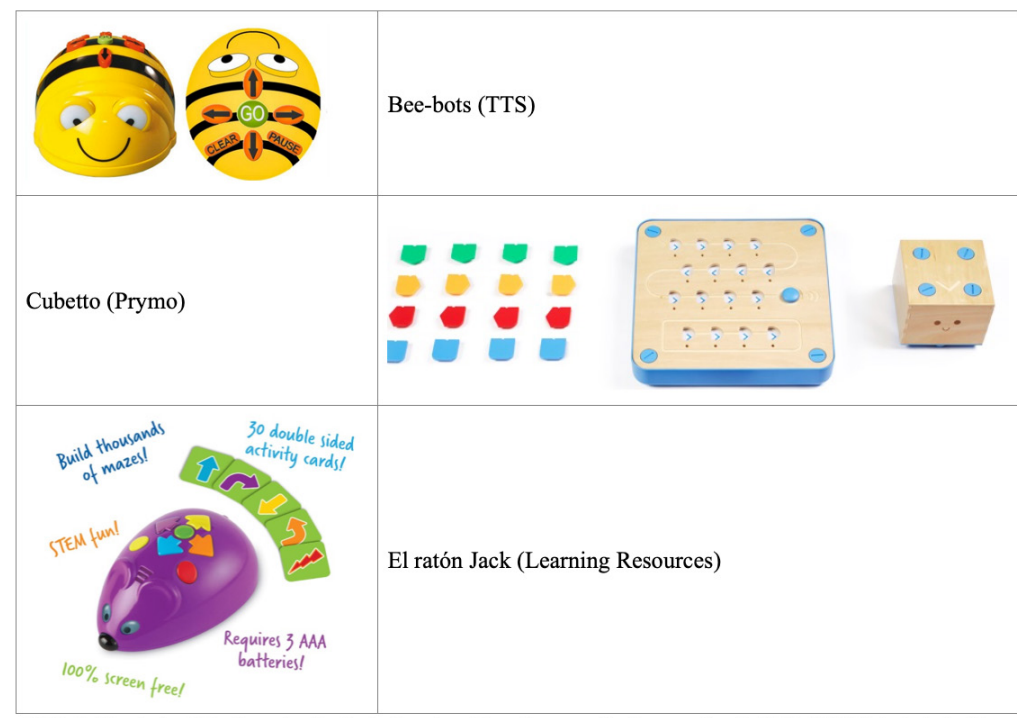

Tabla 1. Bee-bots, Cubetto y el ratón Jack. Fuentes:

https://www.ro-botica.com/tienda/BEE-BOT/Robots-infantiles-programables-TTS/ (Bee-bots), https://cubetto.vicensvives.com/ (Cubetto), https://www.learningresources.com/stem-robot-mouse (Jack) 
Se trata de propuestas orientadas específicamente a 3-6 años, pues no precisan de que el alumnado sepa leer, solamente requieren de la comprensión de los símbolos y la conexión de esta simbología con el entorno. Se trata de propuestas manipulativas que invitan a un aprendizaje a partir del juego exploratorio. En todas ellas, las matemáticas aparecen desde los contenidos: razonamiento lógico (patrones, correspondencias, secuencias temporales), numeración (conteo), geometría (lateralidad, orientación espacial); y, desde los procesos matemáticos: la comunicación de ideas matemáticas, la resolución de problemas, las conexiones, la representación y/o el razonamiento (NCTM, 2000).

En Educación Infantil existen otras propuestas para introducir a las niñas y niños en el mundo de la codificación, con actividades concretas para edades de 5 a 7 años como el ScratchJr (https://www.scratchjr.org/), inspirado en el lenguaje de programación Scratch pensado a partir de los 8 años. El ScratchJr es un lenguaje de programación compuesto por "bloques de programación" diseñados para parecer piezas de rompecabezas con propiedades visuales que corresponden a sus propiedades sintácticas.

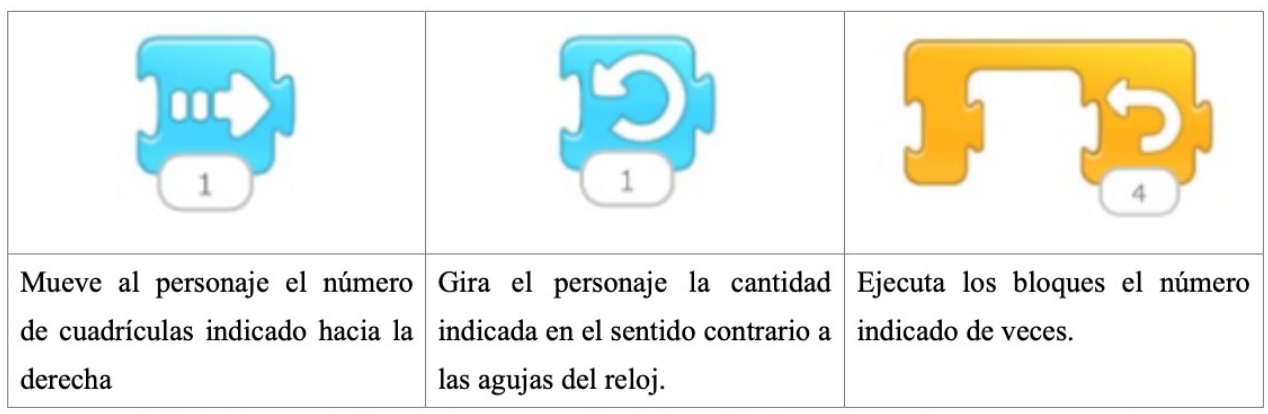

Tabla 2. Muestra de bloques de programación de ScratchJr. Fuente: https://www.scratchjr.org/.

El ScratchJr emplea, como el Beebot, el Cubetto o el ratón Jack, simbología próxima a las ideas que representa, pero en cambio, tiene como punto débil el no ser una propuesta manipulativa, algo esencial para el aprendizaje especialmente en las primeras edades. En cuanto al papel de las matemáticas, vemos que es muy similar en todas ellas. Con una fuerte presencia de la representación (por ejemplo, al dibujar los circuitos, la simbología de las acciones), la resolución de problemas (por ejemplo, tomando decisiones sobre las acciones a realizar), el razonamiento lógico (por ejemplo, al entender por qué el Beebot no ha llegado al punto previsto), la numeración (por ejemplo, al contar los avances en cada dirección) y la geometría (especialmente, orientación espacial).

\subsection{Actividades con foco en la construcción y la experimentación}

En este grupo de actividades STEAM encontramos propuestas de construcción (por ejemplo, construir un laberinto con canicas y plastilina) o de experimentación (por ejemplo, a partir del tintado de papel de cocina con colorante alimentario) con la premisa de que todas ellas involucran interdisciplinarmente contenidos STEAM, mediante la resolución de problemas y el trabajo en equipo. 
Se trata de propuestas presentes en libros pensados para familias y personal docente. Estos libros en muchos casos están diseñados para ser fácilmente utilizados por los niños y las niñas, con un formato que apuesta por las imágenes (dejando el texto en plano secundario) para que éstos puedan ver los proyectos y aproximarse a ellos sabiendo cómo se espera que sean (Dziengel, 2018; Scalzo, 2019; entre otros). También pueden encontrarse en repositorios sobre material STEAM (como StemCat, 2020), o en las páginas web de escuelas que apuestan por este tipo de actividades.

Son propuestas que buscan despertar la curiosidad en los niños y niñas para potenciar sus ganas de experimentar, hacer preguntas, predicciones. Son actividades que buscan impulsar un aprendizaje que va más allá de los contenidos al apostar por el pensamiento crítico, la potenciación de la curiosidad y la creatividad, y la resolución de problemas. Sirvan de ejemplo dos posibles propuestas:

- Un laberinto para jugar con canicas. Se trata de una propuesta sencilla que presenta Dziengel (2018) de manera visual, lo que permite al alumno de Educación Infantil, entender fácilmente el objetivo de la propuesta. Requiere utilizar materiales fáciles de encontrar: una caja, plastilina, y una canica. La propuesta para Dziengel (2018) es una actividad de arte (A) e ingeniería (E), que trabaja a partir de la resolución de problemas. Pues en el proceso de diseño será necesario probar y modificar elementos que no funcionen. El niño o niña deberá probar diferentes ubicaciones de las paredes de plastilina y de los parachoques para asegurar que las canicas se mueven como deben. Por lo que se refiere a la dificultad del laberinto será un aspecto que dependerá del diseño.

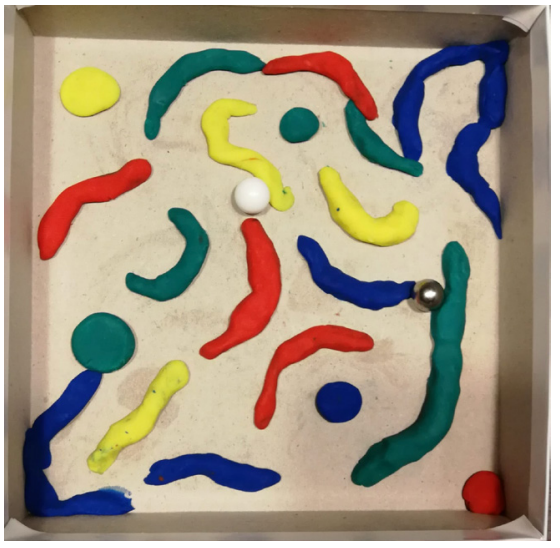

Figura 1. Laberinto de canicas. Fuente: Propia.

- Teñir servilletas. Esta actividad se inspira en las propuestas Tie-Dye Towels (Dziengel, 2018) y Racons d'Art Steam (Sabidó, 2020). Para realizar esta actividad se precisa papel (servilleta, papel de cocina, papel normal); pintura apta para niños; recipientes para la pintura; e instrumentos que permitan pintar (pinceles, pipetas, esponjas). Es una propuesta que pone en juego la $\mathrm{A}$ (arte), la S (ciencias) y la $\mathrm{M}$ (matemáticas). Si el material elegido es el papel de cocina (o la servilleta), les pedimos que la doblen por la mitad, seguidamente, que pongan con la pipeta una gota de cada color una al lado de otra. Lo primero que observarán es cómo los colores 
se mezclan, y al abrir de nuevo la hoja, veremos la imagen simétrica. El niño o la niña descubrirá cómo funciona la capilaridad en una servilleta hecha de celulosa, y esto le permitirá tomar decisiones sobre los colores que quiere combinar y cómo se combinan. Los niños y las niñas pueden preguntarse qué ocurrirá si hacemos más pliegues, o si en vez de plegar la servilleta en dos rectángulos lo hacemos en forma de triángulo. Esta experimentación con la actuación del adulto será lo que aportará complejidad a la actividad.

En cuanto al papel de las matemáticas, se observa en todas las actividades la presencia tanto de los procesos matemáticos (representación, resolución de problemas, conexiones y razonamiento lógico), como de los contenidos matemáticos (especialmente, patrones, numeración, medida y geometría). Así, si revisamos los ejemplos presentados anteriormente vemos que:

- En el caso del laberinto para jugar con canicas, el papel de las matemáticas es esencialmente instrumental. El hecho de que sea así no significa que deje de ser una actividad ideal para promover el aprendizaje de contenido matemático propio de esta etapa, pues permite profundizar en conceptos matemáticos como línea recta o línea curva, circuito abierto o circuito cerrado, la elaboración de "caminos", entre otros. Asimismo, se promueven los procesos matemáticos de resolución de problemas y razonamiento, cuando se diseña el "camino" que recorrerá la canica; la representación del laberinto cuando se elabora el boceto previo en papel; y la comunicación de las ideas matemáticas durante todo el proceso.

- En la propuesta de teñir servilletas, el papel de las matemáticas no es instrumental, pues es uno de los contenidos STEAM que parece tratar la propuesta, al ser una actividad donde se debe plegar un papel por la mitad, entendiéndose como un eje de simetría, para seguidamente observar la imagen simétrica que se obtiene. No obstante, la propuesta solo generará aprendizaje matemático si el adulto explicita las matemáticas que se utilizan. Así, si solo pedimos que plieguen por la mitad sin dar mayor importancia, o al desplegar solo explicitamos lo bonito o mágico que es el dibujo, las matemáticas pasan desapercibidas. En cambio, si el adulto (en nuestro caso la maestra o el maestro) conoce las matemáticas implicadas, sabrá acompañar al alumnado para que vea que, al plegar por la mitad, siempre obtenemos dos imágenes iguales, y, por lo tanto, estaremos ofreciéndoles la oportunidad de experimentar acerca del funcionamiento de las simetrías. En definitiva, la actividad puede tener potencial para trabajar las matemáticas, pero el adulto es el que permite que el aprendizaje se desarrolle.

\subsection{Actividades con foco en el "hazlo tú mismo" (movimiento Maker)}

El movimiento Maker (Do it Yourself) puede concretarse para la educación STEAM integrada en las primeras edades en los makerspaces, entendidos como espacios de creación, espacios físicos donde los niños y las niñas tienen la oportunidad de construir mediante el uso de materiales y herramientas. Espacios que comparten características con los talleres, los estudios de arte y los laboratorios 
de ciencias, y permiten a los creadores idear proyectos que integran las diferentes habilidades de estos espacios (Dougherty, 2013).

Si nos centramos en los makerspace en las escuelas, vemos que para Brejcha (2018) se trataría de un espacio creativo, un espacio físico (y temporal) en el que los alumnos crean, juegan, aprenden a hacer algo nuevo, emprenden desafíos, se divierten, exploran, resuelven problemas, imaginan, construyen, dibujan, escriben, trabajan con las manos, piensan de manera crítica, son persistentes, establecen conexiones con el mundo real y utilizan la tecnología. Así pues, los makerspaces son espacios fluidos, y no hay dos exactamente iguales, pero tienen en común su búsqueda de un aprendizaje para el siglo XXI (Brejcha, 2018).

Los proyectos de makerspace son proyectos creados con material cotidiano, se usan elementos reciclados de todo tipo, desde motores de cepillos dentales eléctricos; a materiales corrientes como cajas, cepillos, recipientes de plástico, tapones, plastilina o globos, entre otros. Se encuentran proyectos que precisan del uso de pequeños motores, y por ello parecen más centrados en Educación Primaria como transformar un cepillo a robot, construir un coche con tapones, una pila y un pequeño motor, circuitos, etc. Pero también existen proyectos para alumnos de Educación Infantil, por ejemplo, construir coches o barcos propulsados por un globo (Graves y Graves, 2016), son proyectos más sencillos y accesibles para las primeras edades.

Figura 2. Barco propulsado por un globo. Fuente: Propia.

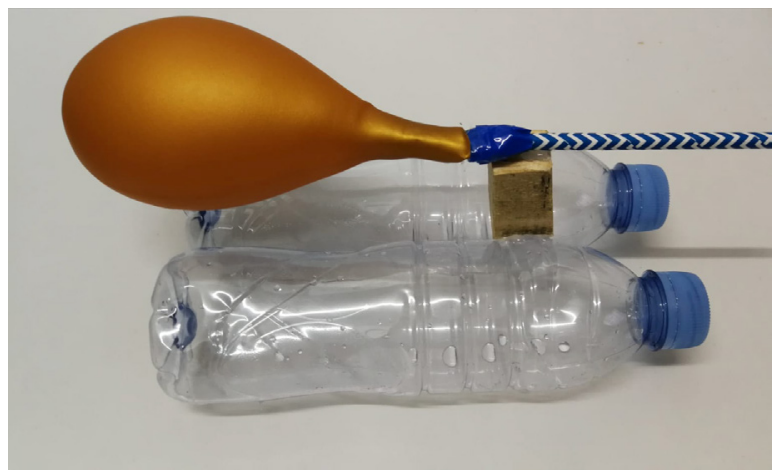

Dentro del movimiento Maker, encontramos la metodología Tinkering que Simarro (2019) traduce por "trastear". El tinkering para algunos autores, como Heroman (2017), sería el pariente lúdico de la ingeniería; pues la ingeniería se inicia a partir de un problema complejo a resolver: "Necesitamos un puente para cruzar a la otra orilla"; mientras, que el Tinkering se inicia a partir de situaciones o problemas más simples, como: "¿Qué puedo hacer con este material?" o "¿Cómo funciona esto?". Para Heroman (2017), tanto en el movimiento Maker, como en la ingeniería o en la metodología Tinkering, el proceso de resolución de problemas es clave; además, en los tres casos se usan materiales y herramientas y se desarrollan las competencias de creatividad y resiliencia. La diferencia está, según Heroman (2017), en que el Tinkering usa cosas ("trastea"), mientras que el Making usa cosas para crear algo (que a veces hacen cosas, o a veces solo son divertidas); y en cambio, la ingeniería "is using stuff to make stuff that does stuff" (Heroman, 2017, p. 4). No obstante, según estos autores el Tinkering y el Maker se usan a menudo indistintamente. 
Podríamos considerar actividades Tinkering aptas para Educación Infantil, después de revisar la literatura, proyectos cómo construir un barco que flote y que pueda transportar a un muñequito tipo lego, diseñar y construir una máscara que se asemeje a un animal para representar un cuento, construir instrumentos musicales (por ejemplo, un tambor con botellas de plástico), etc. (Gabrielson, 2013; Heroman, 2017).

En lo que se refiere a las matemáticas, en la mayoría de los proyectos Maker o Tinkering estas juegan un papel acompañante siendo uno de los instrumentos que permite construir, pero sin restar presencia al proceso de aprendizaje matemático. En este tipo de proyectos aparecen tanto los contenidos como los procesos matemáticos. Así, por ejemplo, estos contenidos aparecen cuando medimos (medida), cuando contamos las piezas que necesitamos (numeración), cuando organizamos en el espacio los distintos elementos (geometría). Y los procesos matemáticos aparecen, por ejemplo: al tomar decisiones acerca del diámetro del tapón adecuado para las ruedas de nuestro coche (resolución de problemas), al dibujar o hacer un croquis del objeto que deseamos construir, o de los pasos que seguiremos (representación), al explicar porque hemos elegido un recipiente u otro para conseguir un sonido más grave o agudo en el tambor (comunicación matemática), y al comprobar si condiciona el tamaño, la forma o la masa de los barcos construidos para que floten (conexiones matemáticas). En definitiva, el potencial de aprendizaje matemático está presente, quien lo hará posible será el docente con la gestión que desarrolle de la actividad.

\section{Aporte de las matemáticas a las actividades STEAM}

Llegados a este punto, nos proponemos dar nuestra visión sobre el papel que ejercen las matemáticas en la educación STEAM integrada en Educación Infantil. El estudio de Simarro y Couso (2018) señala el papel instrumental de las matemáticas en la visión de la educación STEAM de los maestros. Por consiguiente, tiene sentido que desde la didáctica de las matemáticas se haga un esfuerzo para investigar, posicionarse y concretar el papel de las matemáticas en la educación STEAM (Simarro y Couso, 2018), más allá de una visión puramente instrumental de la misma.

Observamos después de revisar distintas actividades STEAM presentes en la etapa de Educación Infantil que el papel que juegan las matemáticas en este tipo de actividades puede ser doble: instrumental o central (en el sentido, de ser una actividad que claramente aborda un problema o reto matemático), de manera similar al papel que juegan las matemáticas en las situaciones del mundo real. Para las matemáticas, ambos tipos de actividades son oportunidades para su aprendizaje, todo dependerá de la gestión que el docente haga de la coyuntura.

Las preguntas del docente para potenciar los aprendizajes matemáticos durante una actividad STEAM son esenciales, tanto si las matemáticas aparecen como instrumento como si tienen un carácter central. Tomemos como ejemplo la actividad del laberinto para jugar con canicas. En dicha actividad pueden plantearse preguntas que permiten aumentar los aprendizajes de los alumnos como: ¿Dónde colocarás la tira de plastilina si quieres que la canica avance? ¿Por qué la canica no ha seguido el camino que querías? ¿Por dónde pasará la canica si la lanzas desde este punto? ¿Qué pasará si inclinas el laberinto a la derecha? ¿Qué camino seguirá la canica roja para llegar a este otro punto? ¿Cuántos caminos diferentes puede seguir la canica? ¿Qué tira de plastilina cambiarías para que la canica pasara por otro lugar? 
En las actividades STEAM integradas, se pone de manifiesto que las matemáticas en Educación Infantil son mucho más que aprender a contar. El pensamiento matemático incluye comparar, clasificar, trabajar con patrones o identificar formas. En esta etapa, el lenguaje tiene también una fuerte importancia en el pensamiento matemático, por ejemplo, al usar las palabras relativas a la comparación (más alto, más cerca, más bajo, etc.). Las actividades STEAM pueden ayudar a los niños y niñas a alcanzar un pensamiento matemático más complejo cuando estos y estas con la ayuda de las actividades que les proponemos son capaces de saber que las comparaciones son relativas, y que algo puede ser más largo o corto en función del objeto con el que comparemos; o, que los objetos pueden ordenarse de distintas maneras (Sharapan, 2012).

En definitiva, las actividades y situaciones STEAM ofrecen un marco ideal para trabajar en Educación Infantil las capacidades matemáticas propias de la etapa: identificar, comparar, clasificar, ordenar, buscar patrones, etc. $Y$ a su vez conseguirlo a partir de los procesos matemáticos, pues son entornos y situaciones que invitan a representar, que necesitan de las conexiones (intra e inter matemáticas), que se desarrollan necesariamente a partir de la resolución de problemas, y donde el razonamiento y la comunicación son necesarios para la elaboración de los proyectos.

\section{Reflexiones finales}

A partir de lo visto en la literatura y en las actividades presentadas, las propuestas STEAM en las aulas de Educación Infantil, sean del tipo que sean, ofrecen a los docentes un espacio interdisciplinar que permite a partir de la resolución de problemas, dar respuesta a un reto, pregunta, situación cotidiana, etc. Son experiencias que a su vez apuestan por una educación que prepare a los futuros ciudadanos con habilidades que se consideran esenciales para el siglo XXI como la creatividad, la colaboración, la comunicación o el pensamiento crítico (Beswick y Fraser, 2019).

Lo cierto es que los estudios consultados sobre la visión que tienen los docentes del aporte de las matemáticas en la educación STEAM, especialmente en las primeras edades, parecen dar a esta disciplina un papel instrumental (Simarro y Couso, 2018). En este trabajo esperamos haber mostrado otra visión, que pone el foco no tanto en la tipología de actividades STEAM que se plantean, sino en la formación de los docentes, inicial y continua. Consideramos que las matemáticas, en todas las propuestas STEAM, sea cual sea su tipología, ofrecen al docente una excusa, un reto o una realidad concreta para que puedan abordar conceptos matemáticos propios de la etapa con sus alumnos, más allá de un uso instrumental de los mismos.

Así pues, más que la presencia de las matemáticas en las actividades STEAM en Educación Infantil, consideramos que tenemos que preguntarnos acerca de la formación de los docentes de Educación Infantil en matemáticas. Preguntarnos desde la didáctica de las matemáticas, cómo debemos preparar a los futuros docentes de Educación Infantil, para la realidad de las aulas que apuestan por propuestas STEAM integradas. Una realidad que demanda docentes que sepan interconectar los contenidos, que sepan distinguir y potenciar cada materia. Probablemente, mientras en los grados de Educación Infantil no haya ninguna asignatura que aborde de manera clara, la educación STEAM integrada deberemos ser los docentes de las asignaturas de didáctica de las matemáticas, los que deberemos dedicar tiempo para abordar en nuestras aulas ejemplos de actividades STEAM como las 
presentadas; con el objetivo de que los alumnos de los grados de Educación Infantil puedan, en su futuro como docentes, obtener el máximo potencial matemático de las actividades STEAM integradas en sus aulas.

\section{Referencias}

Bennett, C.A. y Ruchti, W. (2014). Bridging STEM with mathematical practices. Journal of STEM Teacher Education, 49(1), article 5. https://doi.org/10.30707/JSTE49.1Bennett

Beswick, K. y Fraser, S. (2019). Developing mathematics teachers' 21st century competence for teaching in STEM contexts. ZDM, 51(6), 955-965. https://doi.org/gqsq

Brejcha, L (2018). Makerspaces in school: A month-by-month schoolwide model for building meaningful makerspaces. Prufrock Press Inc.

Caguana, L. G., Alves-Rodrigues, M. I. y Solís, M. C. (2017). Cubetto for preschoolers: Computer programming code to code. En 2017 International Symposium on Computers in Education (SIIE) (pp. 1-5). IEEE.

Catchen, R. (2013). Reflections How STEM becomes STEAM. The STEAM Journal 1(1), art.22. https://doi.org/10.5642/steam.201301.22

Ceschini, J. (2014). STEM + art: A fruitful combination. Education Week, 34(13), 22-23. https://www.edweek.org/ew/articles/2014/12/03/13ceschini.h34.html

DeJarnette, N. K. (2018). Implementing STEAM in the early childhood classroom. European Journal of STEM Education, 3(3), 18. https://doi.org/10.20897/ejsteme/3878

Domènech-Casal, J. (2018). Aprendizaje Basado en Proyectos en el marco STEM. Componentes didácticas para la competencia científica. Ápice. Revista De Educación Científica, 2(2), $29-42$. https://doi.org/10.17979/arec.2018.2.2.4524

Dougherty, D. (2013). The Maker mindset. En M. Honey (Ed.), Design, make, play: Growing the next generation of STEM innovators (pp. 7-11). Taylor \& Francis.

Dziengel, A. (2018). STEAM. Play \& learn. Walter Foster, Jr.

Gabrielson, C. (2013). Tinkering: Kids learn by making stuff. Safari Books.

Graves, C. y Graves, A. (2016) The big book of Makerspace projects: Inspiring makers to experiment, create, and learn. McGraw Hill.

English, L. D. (2016). STEM education K-12: Perspectives on integration. International Journal of STEM education, 3(1), 3. https://doi.org/10.1186/s40594-016-0036-1

Ferrada, C., Díaz-Levicoy, D., Salgado-Orellana, N. y Parraguez, R. (2019). Propuesta de actividades STEM con Bee-bot en matemática. Edma o-6: Educación Matemática en la Infancia, 8(1), 33-43. https://www.edmao-6.es/index.php/edmao-6/article/view/72

Heroman, C. (2017). Making \& tinkering with STEM. Solving design challenges with young children. NAEYC Books.

Holmlund, T. D., Lesseig, K. y Slavit, D. (2018). Making sense of "STEM education" in K-12 contexts. International Journal of STEM Education, 5, 32. https://doi.org/ghg8cm

Jamil, F. M., Linder, S. M. y Stegelin, D. A. (2018). Early childhood teacher beliefs about STEAM education after a professional development conference. Early Childhood Education Journal, 46, 409-417. https://doi.org/10.1007/s10643-017-0875-5

Kelley, T. R. y Knowles, J. G. (2016). A conceptual framework for integrated STEM education. International Journal of STEM Education, 3, 11. https://doi.org/ggwzzs 
McClure, E. R., Guernsey, L., Clements, D. H., Bales, S. N., Nichols, J., Kendall-Taylor, N. y Levine, M. H. (2017). STEM starts early: Grounding science, technology, engineering, and math education in early childhood. The Joan Ganz Cooney Center at Sesame Workshop.

NAENRC - National Academy of Engineering and National Research Council. (2014). STEM integration in K-12 education: Status, prospects, and an agenda for research. The National Academies Press. https://doi.org/10.17226/18612

NCTM -National Council of Teachers of Mathematics (2000). Principios y estándares para la educación matemática. SAEM Thales.

Papadakis, S., Kalogiannakis, M. y Zaranis, N. (2016). Developing fundamental programming concepts and computational thinking with ScratchJr in preschool education: a case study. International Journal of Mobile Learning and Organisation, 1O(3), 187-202.

https://doi.org/10.1504/IJMLO.2016.077867

Portelance, D. J., Strawhacker, A. L. y Bers, M. U. (2016). Constructing the ScratchJr programming language in the early childhood classroom. International Journal of Technology and Design Education, 26(4), 489-504. https://doi.org/10.1007/s10798-015-9325-0

Rennie, L., Venville, G. y Wallace, J. (2017). Making STEM curriculum useful, relevant, and motivating for students. En R. Jorgensen y R. Larkin (Eds.), STEM education in the Junior Secondary (pp. 91-109). Springer.

Russo, M., Hecht, D., Burghardt, M. D., Hacker, M. y Saxman, L. (2011). Development of multidisciplinary middle school mathematics infusion model. Middle Grades Research Journal, 6(2), 113-128. https://bit.ly/3xu2quD

Sabidó, C. (2020). Racons Art STEAM. https://bit.ly/3jw2NzB

Sanders, M. (2009) STEM, STEM education, STEMmania. The Technology Teacher, 68(4), 20-26. https://www.teachmeteamwork.com/files/sanders.istem.ed.ttt.istem.ed.def.pdf

Scalzo, A. (2019). 100 Easy STEAM activities. Page Street Publishing Co.

Sharapan, H. (2012). From STEM to STEAM: How early childhood educators can apply Fred Rogers' approach. Young Children, 67(1), 36-40.

Simarro, C. (2019). El paper del Tinkering en la educación STEM no formal. (Tesis doctoral, Universitat Autònoma de Barcelona). https://hdl.handle.net/10803/667284

Simarro, C. y Couso, D. (2018). Visiones en educación STEAM: y las mates, ¿̇qué?. Uno: Revista de Didáctica de las Matemáticas, 81, 49-56. https://bit.ly/3rVt1Q9

SteamCat (2020). https://projectes.xtec.cat/steamcat/general/propostes-steam-formacions/

Yakman, G. y Lee, H. (2012). Exploring the exemplary STEAM Education in the U.S. as a practical educational framework for Korea. Journal of the Korean Association for Science Education, 32(6), 1072-1086. https://doi.org/10.14697/jkase.2012.32.6.1072 